\title{
Fast Spectral Collocation Method for Solving Nonlinear Time-Delayed Burgers-Type Equations with Positive Power Terms
}

\author{
A. H. Bhrawy, ${ }^{1,2}$ L. M. Assas, ${ }^{1,3}$ and M. A. Alghamdi ${ }^{1}$ \\ ${ }^{1}$ Department of Mathematics, Faculty of Science, King Abdulaziz University, Jeddah 21589, Saudi Arabia \\ ${ }^{2}$ Department of Mathematics, Faculty of Science, Beni-Suef University, Beni Suef 62511, Egypt \\ ${ }^{3}$ Department of Mathematics, Faculty of Science, Um-Al-Qurah University, Makkah 21955, Saudi Arabia
}

Correspondence should be addressed to A. H. Bhrawy; alibhrawy@yahoo.co.uk

Received 20 January 2013; Revised 6 June 2013; Accepted 6 June 2013

Academic Editor: Mustafa Bayram

Copyright (C) 2013 A. H. Bhrawy et al. This is an open access article distributed under the Creative Commons Attribution License, which permits unrestricted use, distribution, and reproduction in any medium, provided the original work is properly cited.

Since the collocation method approximates ordinary differential equations, partial differential equations, and integral equations in physical space, it is very easy to implement and adapt to various problems, including variable coefficient and nonlinear differential equations. In this paper, we derive a Jacobi-Gauss-Lobatto collocation method (J-GL-C) to solve numerically nonlinear timedelayed Burgers-type equations. The proposed technique is implemented in two successive steps. In the first one, we apply $(N-1)$ nodes of the Jacobi-Gauss-Lobatto quadrature which depend upon the two general parameters $(\theta, \vartheta>-1)$, and the resulting equations together with the two-point boundary conditions constitute a system of $(N-1)$ ordinary differential equations (ODEs) in time. In the second step, the implicit Runge-Kutta method of fourth order is applied to solve a system of $(N-1)$ ODEs of second order in time. We present numerical results which illustrate the accuracy and flexibility of these algorithms.

\section{Introduction}

Spectral methods have emerged as powerful techniques used in applied mathematics and scientific computing to numerically solve linear and nonlinear differential equations [1-4] and integral equations [5-7]. Also, they have become increasingly popular for solving fractional differential equations [810]. The main idea of spectral methods is to put the solution of the problem as a sum of certain basic functions and then to choose the coefficients in the sum in order to minimize the difference between the exact solution and the approximate one as well as possible. The choice of test functions leads to the three well-known types of spectral methods, namely, the Galerkin, tau, and collocation methods [11-14]. Spectral collocation method has an exponential convergence rate, which is very useful in providing highly accurate solutions to nonlinear differential equations even using a small number of grids.

The Jacobi polynomials satisfy the orthogonality condition on the interval $[-1,1]$ with respect to the weight function $(1-x)^{\theta}(l+x)^{9}$. There are many special cases of the Jacobi polynomials such as Gegenbauer, Legendre, Zernike, ultraspherical, and Chebyshev polynomials [15]. In recent decades, the use of Jacobi polynomials for solving differential equations has gained increasing popularity due to obtaining the solution in terms of the Jacobi parameters $\theta$ and $\vartheta$ (see, e.g., $[16,17])$.

Time-delay partial differential equations are a type of differential equations in which the derivative of the unknown function at a certain time is given in terms of the values of the function at previous times. They have a wide range of applications in science and engineering such as physical, chemical, and biological sciences $[18,19]$. Also, time-delayed nonlinear partial differential equations described the propagation and transport phenomena or population dynamics.

The solution of delay differential equations has been presented by many authors, but we briefly review some of them. In [20], Ghasemi and Kajani developed a numerical approach of time-varying delay systems using Chebyshev wavelets. Hybrid functions together with Legendre polynomials are investigated in [21-23] to obtain efficient numerical solution of delay systems. Sedaghat et al. [24] presented a numerical 
scheme based on Chebyshev polynomials to treat the delay differential equations of pantograph type. The authors in [25] proposed a Bernoulli operational matrix method for solving generalized pantograph equation. Recently, Ali et al. [26] implemented spectral Legendre approach for solving pantograph-type differential and integral equations with studying the error analysis of the method. More recently, the work of Trif [27] discussed the application of the Tau method based on operational matrix of Chebyshev polynomials for solving DDEs of pantograph-type.

Recently, the authors of [28] and [29] presented some new travelling wave solutions of Burgers equation with finite transport memory and the Korteweg-de Vries-Burgers equation, respectively. Pandey et al. [30] investigated Du Fort-Frankel finite difference approach for solving Burgers equation in finite domain. Meanwhile, in [31], Sun and $\mathrm{Wu}$ presented and applied an efficient numerical solution for the Burgers equation based on a difference scheme in an unbounded domain. More recently, a differential quadrature scheme for Burgers equations was proposed in [32]. The idea of commutative hypercomplex mathematics and the homotopy perturbation method were combined to investigate solutions of time-delayed Burgers equation by Rostamy and Karimi [33]. The Darboux transformation was described in [34] to determine the exact solutions to the Burgers equation. Caglar and Ucar [35] proposed the nonpolynomial cubic spline scheme to develop a numerical solution of Burgers equation. Regarding the two-dimensional Burgers equation, Wang and Zhao [36] presented a novel combination of two-dimensional Haar wavelet functions based on tensorial products for solving two-dimensional Burgers equation.

Time-delayed Burgers equation has a wide range of application in many areas of applied sciences, for example, forest fire, population growth models, and Neolithic transitions [37, 38]. The time-delayed Burgers-Fisher equation is a very important model to forest fire, population growth, Neolithic transitions, the interaction between the reaction mechanism, convection effect and diffusion transport, and so forth [37]. Fahmy et al. [39] used improved tanh function, variational iteration, and the Adomian decomposition methods to present some exact solutions and numerical solutions of the time-delayed Burgers equation. With the aid of a subsidiary high-order ODE, Zhang et al. [37] obtained the exact solutions of the generalized time-delayed Burgers-Fisher equation with positive fractional power terms. Jawad et al. [40] introduced the exact solution of time-delayed Burgers equation using complex tanh method. Moreover, $\left(G^{\prime} / G\right)$ expansion method is applied by Kim and Sakthivel [41] to find the exact solutions of time-delayed Burgers equation.

The goal of this paper is to propose an orthogonal collocation scheme for solving three nonlinear time-delay partial differential equations based on Jacobi family in which the roots of the Jacobi orthogonal polynomials whose distributions can be tuned by two parameters $\theta$ and $\vartheta$. Firstly, we apply the Jacobi-Gauss-Lobatto collocation (J-GL-C) method to the model equation for discretizing spatial derivatives, using $(N-1)$ nodes of the Jacobi-Gauss-Lobatto quadrature which depends upon the two general parameters $(\theta, \vartheta>-1)$; these equations together with the two-point boundary conditions constitute system of $(N-1)$ ordinary differential equations (ODEs) in time. Secondly, the Runge-Kutta method of fourth order is investigated for the time integration of the resulting system of $(N-1)$ nonlinear second-order ODEs.

Indeed, the main advantage of the proposed technique is that the Legendre and Chebyshev collocation methods and other methods can be obtained as special cases from our proposed technique. Comparison of the results obtained by various choices of Jacobi parameters $\theta$ and $\vartheta$ reveals that the present method is very effective and convenient for all choices of $\theta$ and $\vartheta$. Finally, the accuracy of the proposed method is showed by test problems. From the results, these algorithms are extremely efficient and accurate for solving nonlinear time-delayed Burgers'-type equations.

The rest of this paper is structured as follows. In the next section, some basic properties of Jacobi polynomials, which are required in our paper, are given. Section 3 is devoted to the development of Gauss-Lobatto collocation technique for a general form of time-delay partial differential equation based on the Jacobi polynomials, and in Section 4 the proposed method is applied to obtain some numerical results for three problems of time-delay partial differential equations with known exact solutions. Finally, a brief conclusion and some remarks are provided in Section 5.

\section{Preliminaries}

In this section, we briefly recall some properties of the Jacobi polynomials $\left(J_{k}^{(\theta, \vartheta)}(x), k=0,1, \ldots \theta>-1, \vartheta>-1\right)$, which are satisfying the following relations:

$$
\begin{gathered}
J_{k}^{(\theta, 9)}(-x)=(-1)^{k} J_{k}^{(\theta, 9)}(x), \\
J_{k}^{(\theta, 9)}(-1)=\frac{(-1)^{k} \Gamma(k+\vartheta+1)}{k ! \Gamma(\vartheta+1)}, \\
J_{k}^{(\theta, 9)}(1)=\frac{\Gamma(k+\theta+1)}{k ! \Gamma(\theta+1)} .
\end{gathered}
$$

The $q$ th derivative of Jacobi polynomials of degree $k\left(J_{k}^{(\theta, 9)}(x)\right)$ can be given by

$$
D^{(q)} J_{k}^{(\theta, 9)}(x)=\frac{\Gamma(j+\theta+\vartheta+q+1)}{2^{q} \Gamma(j+\theta+\vartheta+1)} J_{k-q, \vartheta+q)}^{(\theta+q, 9}(x .
$$

These polynomials are the only polynomials arising as eigenfunctions of the following singular Sturm-Liouville equation:

$$
\begin{gathered}
\left(1-x^{2}\right) \phi^{\prime \prime}(x)+[\vartheta-\theta+(\theta+\vartheta+2) x] \phi^{\prime}(x) \\
+n(n+\theta+\vartheta+1) \phi(x)=0 .
\end{gathered}
$$

Let $w^{(\theta, 9)}(x)=(1-x)^{\theta}(1+x)^{9}$; then we define the weighted space $L_{w^{(\theta, 9)}}^{2}$ as usual. The inner product and the norm of $L_{w^{(\theta, 9)}}^{2}$ with respect to the weight function are defined as follows:

$$
\begin{gathered}
(u, v)_{w^{(\theta, 9)}}=\int_{-1}^{1} u(x) v(x) w^{(\theta, 9)}(x) d x, \\
\|u\|_{w^{(\theta, 9)}}=(u, u)_{w^{(\theta, 9)}}^{1 / 2} .
\end{gathered}
$$


The set of Jacobi polynomials forms a complete $L_{w^{(\theta, 9)}}^{2}$ orthogonal system, and

$$
\begin{aligned}
\left\|J_{k}^{(\theta, 9)}\right\|_{w^{(\theta, 9)}} & =h_{k} \\
& =\frac{2^{\theta+\vartheta+1} \Gamma(k+\theta+1) \Gamma(k+\vartheta+1)}{(2 k+\theta+\vartheta+1) \Gamma(k+1) \Gamma(k+\theta+\vartheta+1)} .
\end{aligned}
$$

\section{Jacobi Spectral Collocation Method}

The main objective of this section is to develop the J-GL$\mathrm{C}$ method to numerically solve the nonlinear time-delayed Burgers-type equations:

$$
\begin{aligned}
\tau \partial_{t t} v(y, t)+p(1-\tau+q v(y, t)) \partial_{t} v(y, t) \\
=\zeta \partial_{y y} v(y, t)-\lambda(v(y, t))^{s} \\
\quad \times \partial_{y} v(y, t)+q v(y, t)(1-v(y, t)),
\end{aligned}
$$

where

$$
(y, t) \in D \times[0, T], \quad D=\{y: A \leq y \leq B\},
$$

with the boundary-initial conditions

$$
\begin{gathered}
v(A, t)=g_{1}(t), \quad v(B, t)=g_{2}(t), \\
v(y, 0)=f_{1}(y), \quad \partial_{t} v(y, 0)=f_{2}(y), \quad y \in D,
\end{gathered}
$$

where $p, q, s$, and $\lambda$ are real numbers and $\tau>0$ is the time delay. Now, suppose that the change of variables $x=(2 /(B-$ A)) $y+(A+B) /(A-B), u(x, t)=v(y, t)$, which will be used to transform problem (6)-(9) into another one in the classical interval $[-1,1]$ for the space variable, to directly implement collocation method based on Jacobi family defined in $[-1,1]$,

$$
\begin{aligned}
\tau \partial_{t t} u(x, t)+p(1-\tau+q u(x, t)) \partial_{t} u(x, t) \\
=\zeta\left(\frac{2}{B-A}\right)^{2} \partial_{x x} u(x, t)-\lambda(u(x, t))^{s} \\
\quad \times\left(\frac{2}{B-A}\right) \partial_{x} u(x, t)+q u(x, t)(1-u(x, t)),
\end{aligned}
$$

where

$$
(x, t) \in D^{*} \times[0, T], \quad D^{*}=\{x:-1 \leq x \leq 1\},
$$

with the boundary-initial conditions

$$
\begin{gathered}
u(-1, t)=g_{1}(t), \quad u(1, t)=g_{2}(t), \\
u(x, 0)=f_{3}(x), \quad \partial_{t} u(x, 0)=f_{4}(x), \quad x \in D^{*} .
\end{gathered}
$$

The aim of this work is to consider the advantage of the collocation point distribution in a specified domain $[-1,1]$ using the roots of the Jacobi orthogonal polynomials whose distributions can be tuned by two parameters, $\theta$ and $\vartheta$. Now, we outline the main step of the J-GL-C method for solving nonlinear time-delayed Burgers-type equation. Let us expand the dependent variable in a Jacobi series,

$$
u(x, t)=\sum_{j=0}^{N} a_{j}(t) J_{j}^{(\theta, 9)}(x),
$$

and in virtue of (4)-(5), we deduce that

$$
a_{j}(t)=\frac{1}{h_{j}} \int_{-1}^{1} u(x, t) w^{(\theta, \vartheta)}(x) J_{j}^{(\theta, 9)}(x) d x .
$$

To evaluate the previous integral accurately, we present the Jacobi-Gauss-Lobatto quadrature. For any $\phi \in S_{2 N+1}(-1,1)$,

$$
\int_{-1}^{1} w^{(\theta, 9)}(x) \phi(x) d x=\sum_{j=0}^{N} \omega_{N, j}^{(\theta, 9)} \phi\left(x_{N, j}^{(\theta, 9)}\right),
$$

where $S_{N}(-1,1)$ is the set of polynomials of degree less than or equal to $N, x_{N, j}^{(\theta, 9)}(0 \leq j \leq N)$ and $\omega_{N, j}^{(\theta, 9)}(0 \leq j \leq N)$ are the nodes and the corresponding Christoffel numbers of the Jacobi-Gauss-Lobatto quadrature formula on the interval $(-1,1)$, respectively.

In accordance with (15), the coefficients $a_{j}(t)$ in terms of the solution at the collocation points can be approximated by

$$
a_{j}(t)=\frac{1}{h_{j}} \sum_{i=0}^{N} J_{j}^{(\theta, \vartheta)}\left(x_{N, i}^{(\theta, \vartheta)}\right) \omega_{N, i}^{(\theta, \vartheta)} u\left(x_{N, i}^{(\theta, \vartheta)}, t\right) .
$$

Therefore, (13) can be rewritten as

$$
\begin{aligned}
& u(x, t) \\
& =\sum_{i=0}^{N}\left(\sum_{j=0}^{N} \frac{1}{h_{j}} J_{j}^{(\theta, 9)}\left(x_{N, i}^{(\theta, 9)}\right) J_{j}^{(\theta, 9)}(x) \omega_{N, i}^{(\theta, 9)}\right) \\
& \quad \times u\left(x_{N, i}^{(\theta, 9)}, t\right) .
\end{aligned}
$$

Furthermore, if we differentiate (17) once (using (2)) and evaluate it at all Jacobi-Gauss-Lobatto collocation points, it is easy to compute the first spatial partial derivative in terms of the values at these collocation points as

$$
\begin{aligned}
u_{x}\left(x_{N, n}^{(\theta, 9)}, t\right)=\sum_{i=0}^{N}\left(\sum_{j=0}^{N} \frac{j+\theta+\vartheta+1}{2 h_{j}} J_{j}^{(\theta, \vartheta)}\right. & \\
& \left.\times\left(x_{N, i}^{(\theta, 9)}\right) J_{j-1}^{(\theta+1, \vartheta+1)}\left(x_{N, n}^{(\theta, 9)}\right) \omega_{N, i}^{(\theta, \vartheta)}\right) \\
& \times u\left(x_{N, i}^{(\theta, 9)}, t\right), \quad n=0,1, \ldots, N,
\end{aligned}
$$

or it can be shortened to

$$
\begin{array}{r}
u_{x}\left(x_{N, n}^{(\theta, 9)}, t\right)=\sum_{i=0}^{N} A_{n i} u\left(x_{N, i}^{(\theta, 9)}, t\right), \\
n=0,1, \ldots, N,
\end{array}
$$


where

$$
\begin{aligned}
A_{n i}=\sum_{j=0}^{N} & \frac{j+\theta+\vartheta+1}{2 h_{j}} J_{j}^{(\theta, 9)} \\
& \times\left(x_{N, i}^{(\theta, 9)}\right) J_{j-1}^{(\theta+1,9+1)}\left(x_{N, n}^{(\theta, 9)}\right) \omega_{N, i}^{(\theta, 9)} .
\end{aligned}
$$

Similar steps can be applied to the second spatial partial derivative to get

$$
\begin{gathered}
u_{x x}\left(x_{N, n}^{(\theta, 9)}, t\right) \\
=\sum_{i=0}^{N}\left(\sum_{j=0}^{N} \frac{(j+\theta+\vartheta+2)(j+\theta+\vartheta+1)}{4 h_{j}}\right. \\
\left.\times J_{j}^{(\theta, 9)}\left(x_{N, i}^{(\theta, 9)}\right) J_{j-2}^{(\theta+2,9+2)}\left(x_{N, n}^{(\theta, 9)}\right) \omega_{N, i}^{(\theta, 9)}\right) \\
\times u\left(x_{N, i}^{(\theta, 9)}, t\right)=\sum_{i=0}^{N} B_{n i} u\left(x_{N, i}^{(\theta, 9)}, t\right), \\
n=0,1, \ldots, N,
\end{gathered}
$$

where

$$
\begin{aligned}
B_{n i}=\sum_{j=0}^{N} \frac{(j+\theta+\vartheta+2)(j+\theta+\vartheta+1)}{4 h_{j}} J_{j}^{(\theta, \vartheta)} \\
\quad \times\left(x_{N, i}^{(\theta, 9)}\right) J_{j-2}^{(\theta+2, \vartheta+2)}\left(x_{N, n}^{(\theta, 9)}\right) \omega_{N, i}^{(\theta, \vartheta)} .
\end{aligned}
$$

In the proposed Jacobi-Gauss-Lobatto collocation method, the residual of (6) is set to zero at $N-1$ of Jacobi-GaussLobatto points; moreover, the boundary conditions (8) will be enforced at the two collocation points -1 and 1 . Therefore, adopting (19)-(22) enables one to write (6)-(8) in the form

$$
\begin{aligned}
\tau \ddot{u}_{n}(t)+ & p\left(1-\tau+q u_{n}(t)\right) \dot{u}_{n}(t) \\
= & \zeta\left(\frac{2}{B-A}\right)^{2} \sum_{i=0}^{N} B_{n i} u_{i}(t) \\
& -\lambda\left(\frac{2}{B-A}\right) u_{n}(t)^{s} \sum_{i=0}^{N} A_{n i} u_{i}(t) \\
& +q u_{n}(t)\left(1-u_{n}(t)\right),
\end{aligned}
$$

where

$$
\begin{aligned}
u_{k}(t)=u\left(x_{N, k}^{(\theta, 9)}, t\right), & k=1, \ldots, N-1, \\
n & =1, \ldots, N-1 .
\end{aligned}
$$

This provides an $(N-1)$ system of second-order ordinary differential equations in the expansion coefficients $a_{j}(t)$, namely,

$$
\begin{aligned}
\tau \ddot{u}_{n}(t)+ & p\left(1-\tau+q u_{n}(t)\right) \dot{u}_{n}(t) \\
= & \zeta\left(\frac{2}{B-A}\right)^{2}\left(\sum_{i=1}^{N-1} B_{n i} u_{i}(t)+\tilde{d}_{n}(t)\right) \\
& +q u_{n}(t)\left(1-u_{n}(t)\right)-\lambda\left(\frac{2}{B-A}\right) u_{n}(t)^{s} \\
& \times\left(\sum_{i=1}^{N-1} A_{n i} u_{i}(t)+d_{n}(t)\right),
\end{aligned}
$$

where

$$
\begin{aligned}
& d_{n}(t)=A_{n o} g_{1}(t)+A_{n N} g_{2}(t), \\
& \widetilde{d}_{n}(t)=B_{n o} g_{1}(t)+B_{n N} g_{2}(t) .
\end{aligned}
$$

This means that problem (6)-(9) is transformed to the following system of ordinary differential equations (SODEs):

$$
\begin{aligned}
\tau \ddot{u}_{n}(t)+ & p\left(1-\tau+q u_{n}(t)\right) \dot{u}_{n}(t) \\
= & \zeta\left(\frac{2}{B-A}\right)^{2}\left(\sum_{i=1}^{N-1} B_{n i} u_{i}(t)+\widetilde{d}_{n}(t)\right) \\
& +q u_{n}(t)\left(1-u_{n}(t)\right) \\
& -\lambda\left(\frac{2}{B-A}\right) u_{n}(t)^{s}\left(\sum_{i=1}^{N-1} A_{n i} u_{i}(t)+d_{n}(t)\right),
\end{aligned}
$$

subject to the initial values

$$
\begin{gathered}
u_{n}(0)=f_{3}\left(x_{N, n}^{(\theta, 9)}\right), \\
\dot{u}_{n}(0)=f_{4}\left(x_{N, n}^{(\theta, 9)}\right), \quad n=1, \ldots, N-1 .
\end{gathered}
$$

Finally, (27)-(28) can be rewritten into a matrix form of $N-1$ second-order ordinary differential equations with their vectors of initial values:

$$
\begin{aligned}
\ddot{\mathbf{u}}(t)+\dot{\mathbf{u}}(t) & =\mathbf{F}(t, u(t)), \\
\mathbf{u}(0) & =\mathbf{f}_{3}, \\
\dot{\mathbf{u}}(0) & =\mathbf{f}_{4},
\end{aligned}
$$


where

$$
\begin{gathered}
\ddot{\mathbf{u}}(t)=\left[\tau \ddot{u}_{1}(t), \tau \ddot{u}_{2}(t), \ldots, \tau \ddot{u}_{N-1}(t)\right]^{T}, \\
\dot{\mathbf{u}}(t)=\left[p\left(1-\tau+q u_{n}(t)\right) \dot{u}_{1}(t),\right. \\
p\left(1-\tau+q u_{n}(t)\right) \dot{u}_{2}(t), \ldots, \\
\left.p\left(1-\tau+q u_{n}(t)\right) \dot{u}_{N-1}(t)\right]^{T}, \\
\mathbf{f}_{3}=\left[f_{3}\left(x_{N, 1}^{(\theta, 9)}\right), f_{3}\left(x_{N, 2}^{(\theta, 9)}\right), \ldots, f_{3}\left(x_{N, N-1}^{(\theta, 9)}\right)\right]^{T}, \\
\mathbf{f}_{4}=\left[f_{4}\left(x_{N, 1}^{(\theta, 9)}\right), f_{4}\left(x_{N, 2}^{(\theta, 9)}\right), \ldots, f_{4}\left(x_{N, N-1}^{(\theta, 9)}\right)\right]^{T}, \\
\mathbf{F}(t, u(t))=\left[F_{1}(t, u(t)), F_{1}(t, u(t)), \ldots, F_{N-1}(t, u(t))\right]^{T},
\end{gathered}
$$

where

$$
\begin{aligned}
F_{n}(t, u(t)) & \\
= & \zeta\left(\frac{2}{B-A}\right)^{2}\left(\sum_{i=1}^{N-1} B_{n i} u_{i}(t)+\widetilde{d}_{n}(t)\right) \\
& -\lambda\left(\frac{2}{B-A}\right) u_{n}(t)^{s}\left(\sum_{i=1}^{N-1} A_{n i} u_{i}(t)+d_{n}(t)\right) \\
& +q u_{n}(t)\left(1-u_{n}(t)\right) .
\end{aligned}
$$

Remark 1. We can replace the Jacobi polynomials by the Legendre, Chebyshev of the first, second, third, and fourth kinds, or Gegenbauer polynomials and replace the nodes of JacobiGauss-Lobatto quadrature by the Legendre-, Chebyshev-, or Gegenbauer-Gauss-Lobatto quadrature (cf. [42-45]), just by taking the special cases $\theta=\mathcal{\vartheta}=0, \theta=\vartheta=-0.5, \theta=-0.5$, $\vartheta=0.5, \theta=0.5, \vartheta=-0.5$, or $\theta=\vartheta$, respectively, in the resulting system of ordinary differential equations.

The SODEs (29) can be solved by using implicit RungeKutta method of fourth order, which is an important family of implicit and explicit iterative methods for the approximation of solution of system of ordinary differential equations. In the next section, we recall that the difference between the measured or inferred value of approximate solution and its actual value (absolute error) is given by

$$
E(x, t)=|u(x, t)-\widetilde{u}(x, t)|,
$$

where $u(x, t)$ and $\widetilde{u}(x, t)$ are the exact solution and the approximate solution at the point $(x, t)$, respectively. Moreover, the maximum absolute error is given by

$$
M_{E}=\operatorname{Max}\{E(x, t): \forall(x, t) \in D \times[0, T]\} .
$$

\section{Numerical Results and Discussions}

This section considers three numerical examples to demonstrate the accuracy and applicability of the proposed method in the present paper. Comparison of the results obtained by various choices of Jacobi parameters $\theta$ and $\vartheta$ reveals that the present method is very effective and convenient for all choices of $\theta$ and $\vartheta$.

We consider the following three examples.

Example 2. As a first example, we consider the nonlinear time-delayed one-dimensional Burgers equation in the form

$$
\tau u_{t t}+u_{t}+\lambda_{1} u u_{x}-u_{x x}=0, \quad(x, t) \in D \times[0, T],
$$

subject to the initial and boundary values

$$
\begin{aligned}
u(A, t)= & \frac{1}{2}\left(1-\tanh \left(\frac{2 \lambda_{1}}{2\left(4-\tau \lambda^{2}\right)}\left(A-\frac{\lambda_{1}}{2} t\right)\right)\right), \\
u(B, t)= & \frac{1}{2}\left(1-\tanh \left(\frac{2 \lambda_{1}}{2\left(4-\tau \lambda^{2}\right)}\left(B-\frac{\lambda_{1}}{2} t\right)\right)\right), \\
u(x, 0)= & \frac{1}{2}\left(1-\tanh \left(\frac{2 \lambda_{1}}{2\left(4-\tau \lambda^{2}\right)}(x)\right)\right), \quad x \in D . \\
u_{t}(x, 0)= & \frac{\lambda_{1}^{2}}{4\left(4-\tau \lambda^{2}\right)} \\
& \times \operatorname{sech}^{2}\left(\frac{2 \lambda_{1}}{2\left(4-\tau \lambda^{2}\right)}\left(x-\frac{\lambda_{1}}{2} t\right)\right), \quad x \in D .
\end{aligned}
$$

The exact solution [39] of (34) is

$$
u(x, t)=\frac{1}{2}\left(1-\tanh \left(\frac{2 \lambda_{1}}{2\left(4-\tau \lambda^{2}\right)}\left(x-\frac{\lambda_{1}}{2} t\right)\right)\right) .
$$

Maximum absolute errors of (34) subject to (35) and (36) are introduced in Table 1 using J-GL-C method with various choices of $N, \theta$ and $\vartheta$, and $-A=B=10$; this table indicates that the obtained results are very accurate for a small choice of $N$, while the absolute errors of problem (34) are presented in Table 2 for $\theta=\vartheta=1 / 2, \lambda_{1}=0.01, \tau=0.5$, and $N=12$ with different values of $(x, t)$ in the interval $[0,100]$.

In Figure 1, we see that the approximate solution and the exact solution for $t=0.5$ of problem (34) coincide with values of parameters listed in their caption. Moreover, the absolute error of problem (34) where $\theta=\vartheta=-1 / 2$ and $N=2$ is displayed in Figure 2. In Figure 3, we plotted the approximate solution $\widetilde{u}(x, t)$. This assertion shows that the obtained numerical results are accurate and compare favorably with the exact solution.

Example 3. Consider the nonlinear time-delayed onedimensional generalized Burgers equation in the form

$$
\tau u_{t t}+u_{t}+\lambda_{1} u^{s} u_{x}-u_{x x}=0, \quad(x, t) \in D \times[0, T],
$$


TABLE 1: Maximum absolute errors with various choices of $N, \theta$, and $\vartheta$ with $\tau=0.5$ and $\lambda_{1}=0.01$ for Example 2 .

\begin{tabular}{|c|c|c|c|c|c|c|c|c|c|c|c|}
\hline$N$ & $A$ & $B$ & $\theta$ & $\vartheta$ & $M_{E}$ & $N$ & $A$ & $B$ & $\theta$ & $\vartheta$ & $M_{E}$ \\
\hline 2 & -10 & 10 & 0 & 0 & $2.59 \times 10^{-7}$ & 2 & -10 & 10 & 0.5 & 0.5 & $2.59 \times 10^{-7}$ \\
\hline 4 & & & & & $2.70 \times 10^{-11}$ & 4 & & & & & $2.31 \times 10^{-11}$ \\
\hline 6 & & & & & $2.22 \times 10^{-15}$ & 6 & & & & & $1.89 \times 10^{-15}$ \\
\hline 8 & & & & & $1.11 \times 10^{-16}$ & 8 & & & & & $1.11 \times 10^{-16}$ \\
\hline 2 & -10 & 10 & -0.5 & -0.5 & $2.59 \times 10^{-7}$ & 2 & -10 & 10 & -0.5 & 0.5 & $8.95 \times 10^{-7}$ \\
\hline 4 & & & & & $2.23 \times 10^{-11}$ & 4 & & & & & $5.97 \times 10^{-11}$ \\
\hline 6 & & & & & $3.22 \times 10^{-15}$ & 6 & & & & & $3.77 \times 10^{-15}$ \\
\hline 8 & & & & & $1.11 \times 10^{-16}$ & 8 & & & & & $1.11 \times 10^{-16}$ \\
\hline
\end{tabular}

TABLE 2: Absolute errors with $\theta=\vartheta=1 / 2, N=12$ and various choices of $x, t$ with $\tau=0.5$ and $\lambda_{1}=0.01$ for Example 2 .

\begin{tabular}{|c|c|c|c|c|c|c|c|c|c|c|c|}
\hline$x$ & $t$ & $N$ & $A$ & $B$ & $E$ & $x$ & $t$ & $N$ & $A$ & $B$ & $E$ \\
\hline 0 & 0.1 & 12 & 0 & 100 & $3.33 \times 10^{-16}$ & 0 & 0.2 & 12 & 0 & 100 & $7.77 \times 10^{-16}$ \\
\hline 10 & & & & & $5.55 \times 10^{-17}$ & 10 & & & & & $5.55 \times 10^{-17}$ \\
\hline 02 & & & & & $5.55 \times 10^{-17}$ & 20 & & & & & 0 \\
\hline 30 & & & & & $5.55 \times 10^{-17}$ & 30 & & & & & $5.55 \times 10^{-17}$ \\
\hline 40 & & & & & $5.55 \times 10^{-17}$ & 40 & & & & & 0 \\
\hline 50 & & & & & 0 & 50 & & & & & $5.55 \times 10^{-17}$ \\
\hline 60 & & & & & $1.67 \times 10^{-16}$ & 60 & & & & & $5.55 \times 10^{-17}$ \\
\hline 70 & & & & & 0 & 70 & & & & & $5.55 \times 10^{-17}$ \\
\hline 80 & & & & & $1.11 \times 10^{-16}$ & 80 & & & & & 0 \\
\hline 90 & & & & & $1.67 \times 10^{-16}$ & 90 & & & & & 0 \\
\hline 100 & & & & & $5.00 \times 10^{-16}$ & 100 & & & & & $5.55 \times 10^{-17}$ \\
\hline
\end{tabular}

subject to the boundary conditions

$u(A, t)$

$$
=\sqrt[s]{\frac{1}{2}\left(1-\tanh \left(\frac{s(s+1) \lambda_{1}}{2\left((s+1)^{2}-\tau \lambda^{2}\right)}\left(A-\frac{\lambda_{1}}{(s+1)} t\right)\right)\right)},
$$

$u(B, t)$

$$
=\sqrt[s]{\frac{1}{2}\left(1-\tanh \left(\frac{s(s+1) \lambda_{1}}{2\left((s+1)^{2}-\tau \lambda^{2}\right)}\left(B-\frac{\lambda_{1}}{(s+1)} t\right)\right)\right.},
$$

and the initial conditions

$$
u(x, 0)=\sqrt[s]{\frac{1}{2}\left(1-\tanh \left(\frac{s(s+1) \lambda_{1}}{2\left((s+1)^{2}-\tau \lambda^{2}\right)}(x)\right)\right)}
$$

$u_{t}(x, 0)$

$$
\begin{aligned}
& =\frac{s \lambda_{1}^{2} \operatorname{sech}^{2}\left(\left(s(s+1) \lambda_{1} / 2\left((s+1)^{2}-\tau \lambda^{2}\right)\right)(x)\right)}{2\left((s+1)^{2}-\tau \lambda^{2}\right)} \\
& \quad \times \sqrt[s]{\left(\frac{1}{2}\left(1-\tanh \left(\frac{s(s+1) \lambda_{1}}{2\left((s+1)^{2}-\tau \lambda^{2}\right)}(x)\right)\right)\right)^{1-s}},
\end{aligned}
$$

The exact solution [39] of (39) is

$u(x, t)$

$$
=\sqrt[s]{\frac{1}{2}\left(1-\tanh \left(\frac{s(s+1) \lambda_{1}}{2\left((s+1)^{2}-\tau \lambda^{2}\right)}\left(x-\frac{\lambda_{1}}{(s+1)} t\right)\right)\right)} .
$$

Table 3 lists the maximum absolute errors of (39) subject to (40) and (41) in $[-10,10]$, using J-GL-C method for different values of $N, \theta$, and $\vartheta$. Moreover, in Tables 4 and 5 , we evaluate the absolute errors of (39) for $s=3$ and 5 , respectively, with different values of $(x, t)$ in the interval $[-100,100]$.

Figure 4 plots the approximate and the exact solutions at $t=0.5$ of (39) with the special values $\theta=\vartheta=-1 / 2$, 


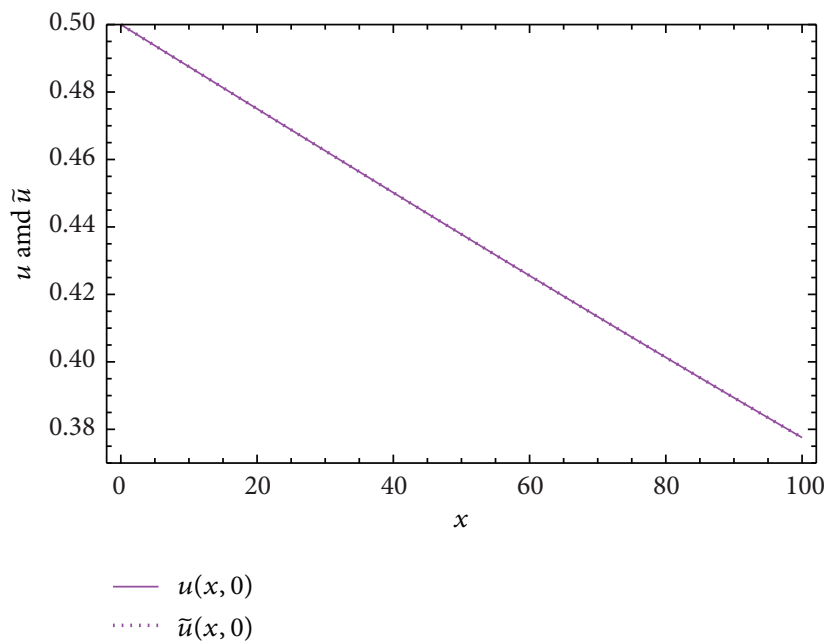

FIGURE 1: The approximate solution and the exact solution for $t=0.5$ of problem (34) with $\theta=\vartheta=1 / 2, \lambda_{1}=0.01, \tau=0.5$, and $N=12$.

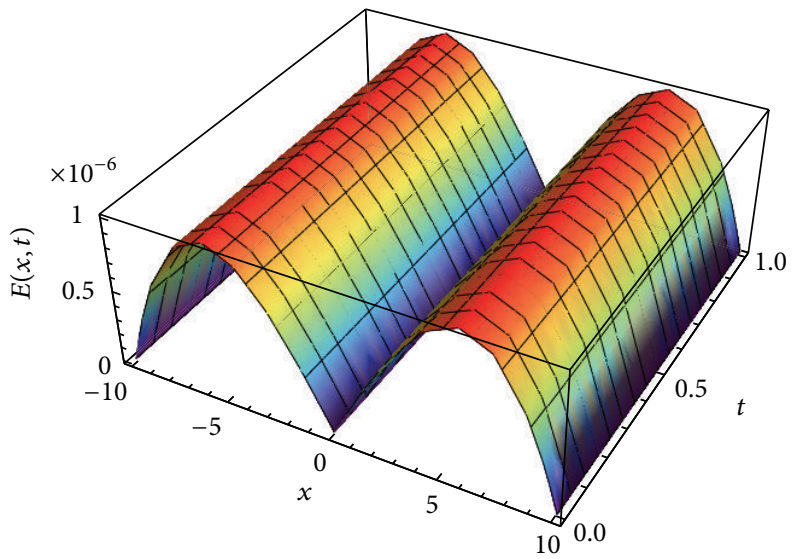

Figure 2: The absolute error between the approximate and the exact solutions of problem (34) with $\theta=\vartheta=-1 / 2, \lambda_{1}=0.01, \tau=0.5$, and $N=2$.

$\lambda_{1}=0.01, s=2$, and $N=12$. Moreover, the absolute errors between the approximate and the exact solutions of (39) with $\theta=\vartheta=1 / 2, \lambda_{1}=0.01, s=3$, and $N=8$ are shown in Figure 5.

Example 4. Consider the nonlinear time-delayed BurgersFisher equation in the form

$$
\begin{array}{r}
\tau u_{t t}+(1-\tau) u_{t}+2 \tau u u_{t} \\
+u u_{x}-u_{x x}-u+u^{2}=0, \\
(x, t) \in D \times[0, T],
\end{array}
$$

subject to the boundary conditions

$$
\begin{aligned}
& u(A, t)=\frac{1}{2}\left(1+\tanh \left(\frac{\tau+1}{\tau-4}\left(A-\frac{5}{2(\tau+1)} t\right)\right)\right) \\
& u(B, t)=\frac{1}{2}\left(1+\tanh \left(\frac{\tau+1}{\tau-4}\left(B-\frac{5}{2(\tau+1)} t\right)\right)\right),
\end{aligned}
$$

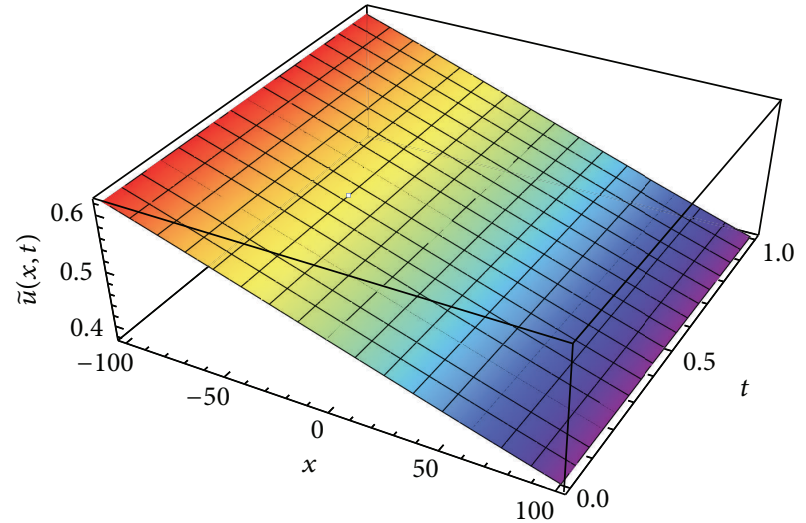

FIGURE 3: The approximate solution of problem (34) with $\theta=9=0$, $\lambda_{1}=0.01, \tau=0.5$, and $N=12$.

and the initial condition

$$
u(x, 0)=\frac{1}{2}\left(1+\tanh \left(\frac{\tau+1}{\tau-4}(x)\right)\right), \quad x \in D,
$$


TABLE 3: Maximum absolute errors with various choices of $N, \theta$, and $\vartheta$ with $\tau=0.5, \lambda_{1}=0.01$, and $s=3$ for Example 3 .

\begin{tabular}{|c|c|c|c|c|c|c|c|c|c|c|c|}
\hline$N$ & $A$ & $B$ & $\theta$ & $\vartheta$ & $M_{E}$ & $N$ & $A$ & $B$ & $\theta$ & $\vartheta$ & $M_{E}$ \\
\hline 2 & -10 & 10 & 0 & 0 & $6.20 \times 10^{-5}$ & 2 & -10 & 10 & 0.5 & 0.5 & $6.20 \times 10^{-5}$ \\
\hline 4 & & & & & $5.53 \times 10^{-9}$ & 4 & & & & & $4.84 \times 10^{-9}$ \\
\hline 6 & & & & & $5.89 \times 10^{-13}$ & 6 & & & & & $4.86 \times 10^{-13}$ \\
\hline 8 & & & & & $2.22 \times 10^{-16}$ & 8 & & & & & $2.22 \times 10^{-16}$ \\
\hline 2 & -10 & 10 & -0.5 & -0.5 & $6.20 \times 10^{-5}$ & 2 & -10 & 10 & -0.5 & 0.5 & $5.202 \times 10^{-5}$ \\
\hline 4 & & & & & $6.45 \times 10^{-9}$ & 4 & & & & & $4.24 \times 10^{-9}$ \\
\hline 6 & & & & & $7.29 \times 10^{-13}$ & 6 & & & & & $4.42 \times 10^{-13}$ \\
\hline 8 & & & & & $3.33 \times 10^{-16}$ & 8 & & & & & $1.11 \times 10^{-16}$ \\
\hline
\end{tabular}

TABLE 4: Absolute errors with $-\theta=\vartheta=1 / 2, N=12$ and various choices of $x, t$ for Example 3 for $\tau=0.5, \lambda_{1}=0.01$, and $s=3$.

\begin{tabular}{lcccccccccc}
\hline$x$ & $t$ & $N$ & $A$ & $B$ & $E$ & $x$ & $t$ & $N$ & $A$ & $B$ \\
\hline-100 & 0.1 & 12 & -100 & 100 & $1.46 \times 10^{-11}$ & -100 & 0.2 & 12 & -100 & 100 \\
-80 & & & & & $1.85 \times 10^{-12}$ & -80 & & & $1.45 \times 10^{-11}$ \\
-60 & & & & & $1.66 \times 10^{-12}$ & -60 & & $1.85 \times 10^{-12}$ \\
-40 & & & & & $1.06 \times 10^{-12}$ & -40 & & $1.16 \times 10^{-12}$ \\
-20 & & & & & $8.93 \times 10^{-13}$ & -20 & & $1.05 \times 10^{-12}$ \\
0 & & & & $4.89 \times 10^{-13}$ & 0 & & $8.92 \times 10^{-13}$ \\
20 & & & & $7.77 \times 10^{-15}$ & 20 & & $4.87 \times 10^{-13}$ \\
40 & & & & $3.54 \times 10^{-13}$ & 40 & & $8.55 \times 10^{-15}$ \\
60 & & & & $4.50 \times 10^{-13}$ & 60 & & $3.54 \times 10^{-13}$ \\
80 & & & & $1.89 \times 10^{-13}$ & 80 & & $4.50 \times 10^{-13}$ \\
100 & & & & $5.83 \times 10^{-13}$ & 100 & & $1.90 \times 10^{-13}$ \\
\hline
\end{tabular}

TABLE 5: Absolute errors with $\theta=\vartheta=-1 / 2, N=12$ and various choices of $x, t$ for Example 3 for $\tau=0.5, \lambda_{1}=0.01$, and $s=5$.

\begin{tabular}{lcccccccccc}
\hline$x$ & $t$ & $N$ & $A$ & $B$ & $E$ & $x$ & $t$ & $N$ & $A$ & $B$ \\
\hline-100 & 0.1 & 12 & -100 & 100 & $2.13 \times 10^{-12}$ & -100 & 0.2 & 12 & -100 & 100 \\
-80 & & & & & $3.24 \times 10^{-13}$ & -80 & & & $2.13 \times 10^{-12}$ \\
-60 & & & & & $2.00 \times 10^{-13}$ & -60 & & & $3.20 \times 10^{-13}$ \\
-40 & & & & & $5.94 \times 10^{-13}$ & -40 & & $2.01 \times 10^{-13}$ \\
-20 & & & & & $1.70 \times 10^{-12}$ & -20 & & $5.92 \times 10^{-13}$ \\
0 & & & & $2.13 \times 10^{-12}$ & 0 & & $1.69 \times 10^{-12}$ \\
20 & & & & $1.46 \times 10^{-12}$ & 20 & & $2.13 \times 10^{-12}$ \\
40 & & & & $2.66 \times 10^{-13}$ & 40 & & $1.45 \times 10^{-12}$ \\
60 & & & & $4.87 \times 10^{-13}$ & 60 & & $2.64 \times 10^{-13}$ \\
80 & & & & $1.13 \times 10^{-13}$ & 80 & & $4.88 \times 10^{-13}$ \\
100 & & & & $2.13 \times 10^{-12}$ & 100 & & $1.08 \times 10^{-13}$ \\
\hline
\end{tabular}

$$
\begin{aligned}
& u_{t}(x, 0)=-\frac{5}{4(\tau-4)} \operatorname{sech}^{2} \\
& \times\left(\frac{\tau+1}{\tau-4}\left(x-\frac{5}{2 \tau+1} t\right)\right), \\
& x \in D .
\end{aligned}
$$

The exact solution [39] of (44) is

$$
u(x, t)=\frac{1}{2}\left(1+\tanh \left(\frac{\tau+1}{\tau-4}\left(x-\frac{5}{2(\tau+1)} t\right)\right)\right) .
$$

Maximum absolute errors of (44) subject to (45) and (46) are tabulated in Table 6 using J-GL-C method with various choices of $N, \theta$, and $\vartheta$, while the absolute errors of (44) are presented in Table 7 for $\theta=\vartheta=0$ and $N=40$ with different values of $(x, t)$ in the interval $[-5,5]$.

In Figure 6, we see that the approximate solution and the exact solution of (44) coincide for different values of $t=0$, 0.5 , and 0.9 and $\theta=\vartheta=0, \lambda_{1}=0.01, \tau=0.5$, and $N=$ 40. The approximate solution of (44) with $\theta=\vartheta=0, \lambda_{1}=$ $0.01, \tau=0.5$, and $N=40$ is displayed in Figure 7, while the absolute error of (44) with $\theta=\vartheta=0, \lambda_{1}=0.01, \tau=0.5$, and $N=40$ is displayed in Figure 8. 
TABLE 6: Maximum absolute errors with various choices of $N, \theta$, and $\vartheta$ with $\tau=0.5$ for Example 4 .

\begin{tabular}{lccccccccccc}
\hline$N$ & $A$ & $B$ & $\theta$ & $\vartheta$ & $M_{E}$ & $N$ & $A$ & $B$ & $\theta$ & $\vartheta$ & $M_{E}$ \\
\hline 8 & -10 & 10 & 0 & 0 & $2.25 \times 10^{-3}$ & 8 & -10 & 10 & 0.5 & 0.5 & $1.92 \times 10^{-3}$ \\
20 & & & & & $6.10 \times 10^{-7}$ & 20 & & & $5.16 \times 10^{-7}$ \\
32 & & & & & $2.83 \times 10^{-10}$ & 32 & & & $3.01 \times 10^{-10}$ \\
\hline 8 & -10 & 10 & -0.5 & -0.5 & $2.73 \times 10^{-3}$ & 8 & -10 & 10 & 0.5 & 0.5 \\
20 & & & & & $7.70 \times 10^{-7}$ & 20 & & & $3.09 \times 10^{-3}$ \\
32 & & & & & $3.36 \times 10^{-10}$ & 32 & & & $8.81 \times 10^{-7}$ \\
\hline
\end{tabular}

TABLE 7: Absolute errors with $\theta=\vartheta=0, N=40$ and various choices of $x, t$ with $\tau=0.5$ for Example 4 .

\begin{tabular}{|c|c|c|c|c|c|c|c|c|c|c|c|}
\hline$x$ & $t$ & $N$ & $A$ & $B$ & $E$ & $x$ & $t$ & $N$ & $A$ & $B$ & $E$ \\
\hline-5 & 0.1 & 12 & -5 & 5 & $4.16 \times 10^{-12}$ & -5 & 0.2 & 12 & -5 & 5 & $1.66 \times 10^{-12}$ \\
\hline-4 & & & & & $1.84 \times 10^{-13}$ & -4 & & & & & $6.58 \times 10^{-13}$ \\
\hline-3 & & & & & $5.07 \times 10^{-13}$ & -3 & & & & & $3.95 \times 10^{-13}$ \\
\hline-2 & & & & & $3.32 \times 10^{-13}$ & -2 & & & & & $5.74 \times 10^{-13}$ \\
\hline-1 & & & & & $2.82 \times 10^{-13}$ & -1 & & & & & $9.45 \times 10^{-13}$ \\
\hline 0 & & & & & $3.59 \times 10^{-13}$ & 0 & & & & & $3.07 \times 10^{-13}$ \\
\hline 1 & & & & & $7.25 \times 10^{-13}$ & 1 & & & & & $2.60 \times 10^{-13}$ \\
\hline 2 & & & & & $1.01 \times 10^{-12}$ & 2 & & & & & $3.42 \times 10^{-13}$ \\
\hline 3 & & & & & $9.92 \times 10^{-13}$ & 3 & & & & & $3.12 \times 10^{-13}$ \\
\hline 4 & & & & & $6.08 \times 10^{-14}$ & 4 & & & & & $7.46 \times 10^{-13}$ \\
\hline 5 & & & & & $4.16 \times 10^{-12}$ & 5 & & & & & $1.66 \times 10^{-12}$ \\
\hline
\end{tabular}

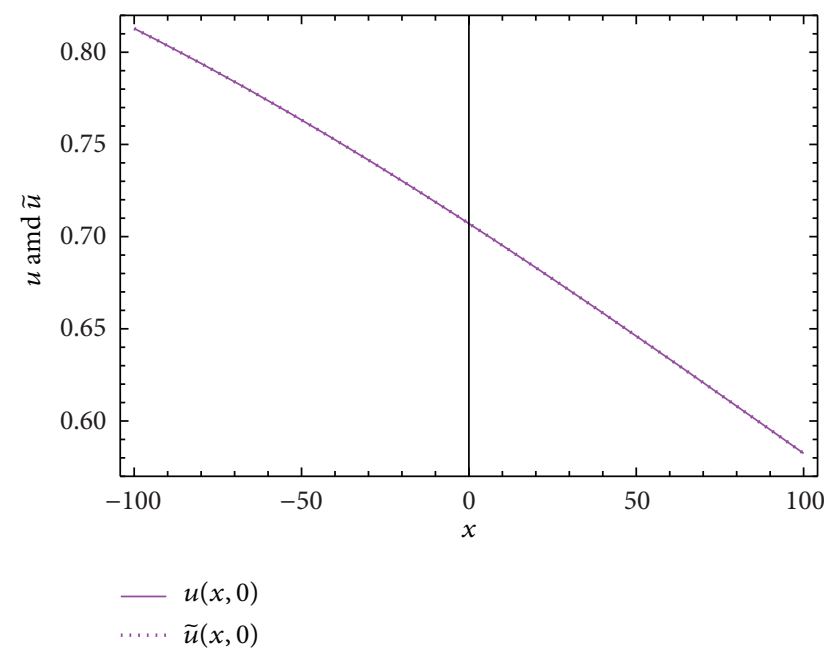

FIGURE 4: The approximate and the exact solutions for different values of $t=0.5$ of problem (39) with $\theta=\vartheta=-1 / 2, \lambda_{1}=0.01$, $s=2$, and $N=12$.

\section{Conclusions and Future Works}

We have constructed in this paper an efficient spectralcollocation algorithm for solving nonlinear time-delayed Burgers-type equations with positive power terms subject to initial and boundary conditions. The Jacobi-Gauss-Lobatto collocation methods based upon two general parameters, $\theta$ and $\vartheta$, are developed and applied to the time-delayed Burgerstype equations for reformulating the problem to a system of second-order ordinary differential equations.

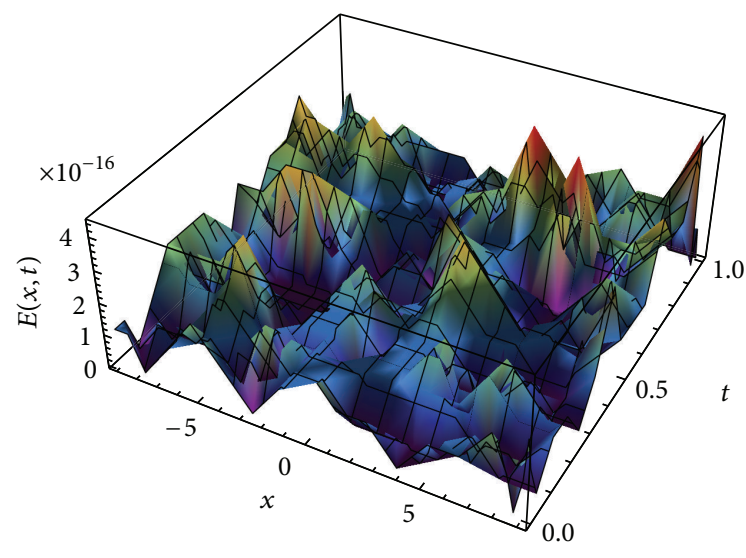

FIgURE 5: The absolute errors between the approximate and the exact solutions of problem (39) with $\theta=\vartheta=1 / 2, \lambda_{1}=0.01, s=3$, and $N=8$.

In fact, Jacobi-Gauss-Lobatto collocation method is slightly more complicated to implement than other orthogonal collocation methods but is more efficient and can be applied to a wider class of problems. We presented some advantages of the presented algorithm, as well as numerical results which demonstrate its accuracy and flexibility.

While the new algorithm presented in this paper only applied to a specific class of nonlinear time-delayed equations, it is very efficient and accurate whenever applicable. Moreover, high accuracy in long computational intervals and the stability of the proposed method encourage us to apply a similar scheme for the numerical solution of coupled 


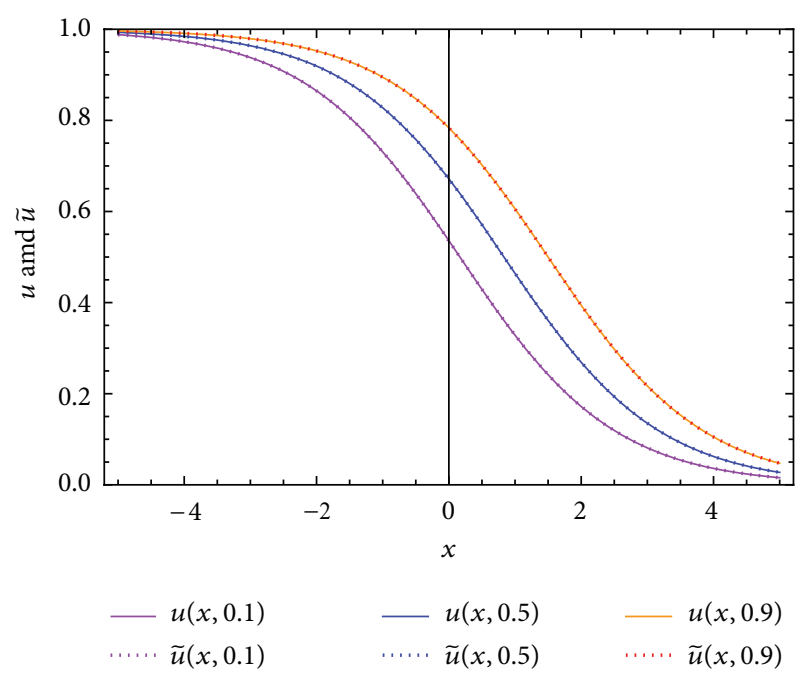

FIgURE 6: The approximate and the exact solutions for different values of $t(0,0.5$, and 0.9$)$ of problem (44) with $\theta=\vartheta=0, \lambda_{1}=0.01$, $\tau=0.5$, and $N=40$.

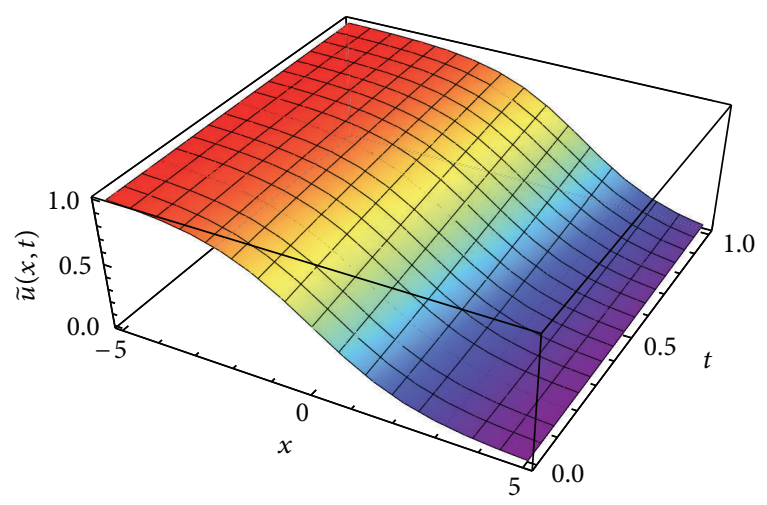

FIgURE 7: The approximate solution of problem (44) with $\theta=\vartheta=0$, $\lambda_{1}=0.01, \tau=0.5$, and $N=40$.

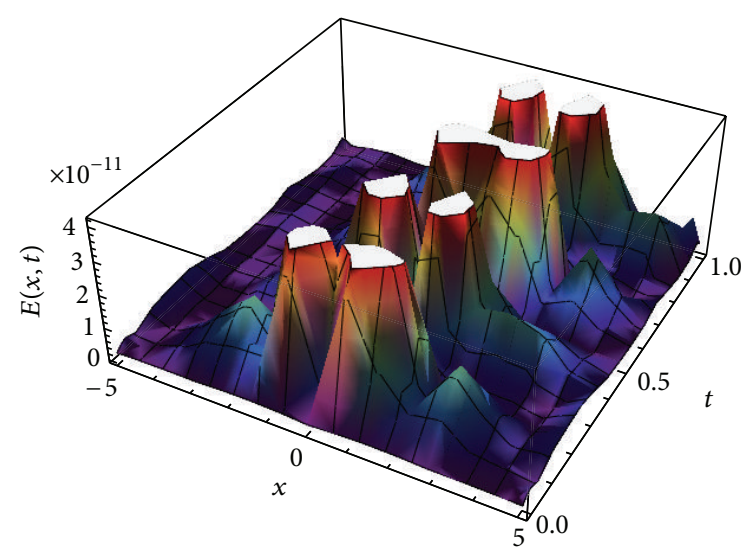

FIGURE 8: The absolute error between the approximate and the exact solutions of problem (44) with $\theta=\vartheta=0, \lambda_{1}=0.01, \tau=0.5$, and $N=40$. nonlinear partial differential equations and other applied mathematics problems (see, e.g, [46-49] and references therein) in the future.

\section{References}

[1] C. Canuto, M. Y. Hussaini, A. Quarteroni, and T. A. Zang, Spectral Methods: Fundamentals in Single Domains, Scientific Computation, Springer, Berlin, Germany, 2006.

[2] C. I. Gheorghiu, Spectral Methods for Differential Problems, T. Popoviciu Institute of Numerical Analysis, Cluj-Napoca, Romaina, 2007.

[3] E. H. Doha, A. H. Bhrawy, and R. M. Hafez, "On shifted Jacobi spectral method for high-order multi-point boundary value problems," Communications in Nonlinear Science and Numerical Simulation, vol. 17, no. 10, pp. 3802-3810, 2012.

[4] E. H. Doha, W. M. Abd-Elhameed, and A. H. Bhrawy, "New spectral-Galerkin algorithms for direct solution of high evenorder differential equations using symmetric generalized Jacobi polynomials," Collectanea Mathematica, 2013.

[5] K. Zhang, J. Li, and H. Song, "Collocation methods for nonlinear convolution Volterra integral equations with multiple proportional delays," Applied Mathematics and Computation, vol. 218, no. 22, pp. 10848-10860, 2012.

[6] S. K. Vanani and F. Soleymani, "Tau approximate solution of weakly singular Volterra integral equations," Mathematical and Computer Modelling, vol. 57, no. 3-4, pp. 494-502, 2013.

[7] L. Zhu and Q. Fan, "Solving fractional nonlinear Fredholm integro-differential equations by the second kind Chebyshev wavelet," Communications in Nonlinear Science and Numerical Simulation, vol. 17, no. 6, pp. 2333-2341, 2012.

[8] E. H. Doha, A. H. Bhrawy, and S. S. Ezz-Eldien, "A Chebyshev spectral method based on operational matrix for initial and boundary value problems of fractional order," Computers \& Mathematics with Applications, vol. 62, no. 5, pp. 2364-2373, 2011.

[9] E. H. Doha, A. H. Bhrawy, and S. S. Ezz-Eldien, "A new Jacobi operational matrix: an application for solving fractional differential equations," Applied Mathematical Modelling, vol. 36, no. 10, pp. 4931-4943, 2012.

[10] D. Rostamy, K. Karimi, L. Gharacheh, and M. Khaksarfard, "Spectral method for fractional quadratic Riccati differential equation," Journal of Applied Mathematics and Bioinformatics, vol. 2, pp. 85-97, 2012.

[11] A. H. Bhrawy and A. S. Alofi, "A Jacobi-Gauss collocation method for solving nonlinear Lane-Emden type equations," Communications in Nonlinear Science and Numerical Simulation, vol. 17, no. 1, pp. 62-70, 2012.

[12] A. H. Bhrawy and M. A. Alghamdi, "A shifted Jacobi-GaussLobatto collocation method for solving nonlinear fractional Langevin equation involving two fractional orders in different intervals," Boundary Value Problems, vol. 2012, article 62, 2012.

[13] K. Maleknejad and M. Attary, "A Chebysheve collocation method for the solution of higher-order Fredholm-Volterra integro-differential equations system," Scientific Bulletin, Universitatea Politehnica din Bucuresti Series A, vol. 74, no. 4, pp. 17-28, 2012.

[14] M. Maleki, I. Hashim, M. T. Kajani, and S. Abbasbandy, "An adaptive pseudospectral method for fractional order boundary value problems," Abstract and Applied Analysis, vol. 2012, Article ID 381708, 19 pages, 2012. 
[15] G. Szegö, Orthogonal Polynomials, Colloquium Publications. XXIII. American Mathematical Society, 1939.

[16] E. H. Doha, A. H. Bhrawy, and R. M. Hafez, "A Jacobi dualPetrov-Galerkin method for solving some odd-order ordinary differential equations," Abstract and Applied Analysis, vol. 2011, Article ID 947230, 21 pages, 2011.

[17] A. H. Bhrawy, E. H. Doha, and R. M. Hafez, "A Jacobi dualPetrov-Galerkin method for solving some odd-order ordinary differential equations," Abstract and Applied Analysis, vol. 2011, Article ID 947230, 16 pages, 2011.

[18] H. Kim and R. Sakthivel, "Travelling wave solutions for timedelayed nonlinear evolution equations," Applied Mathematics Letters, vol. 23, no. 5, pp. 527-532, 2010.

[19] M. Dehghan and R. Salehi, "Solution of a nonlinear time-delay model in biology via semi-analytical approaches," Computer Physics Communications, vol. 181, no. 7, pp. 1255-1265, 2010.

[20] M. Ghasemi and M. T. Kajani, "Numerical solution of timevarying delay systems by Chebyshev wavelets," Applied Mathematical Modelling, vol. 35, no. 11, pp. 5235-5244, 2011.

[21] X. T. Wang, "Numerical solution of delay systems containing inverse time by hybrid functions," Applied Mathematics and Computation, vol. 173, no. 1, pp. 535-546, 2006.

[22] X. T. Wang, "Numerical solutions of optimal control for time delay systems by hybrid of block-pulse functions and Legendre polynomials," Applied Mathematics and Computation, vol. 184, no. 2, pp. 849-856, 2007.

[23] X. T. Wang, "Numerical solutions of optimal control for linear time-varying systems with delays via hybrid functions," Journal of the Franklin Institute, vol. 344, no. 7, pp. 941-953, 2007.

[24] S. Sedaghat, Y. Ordokhani, and M. Dehghan, "Numerical solution of the delay differential equations of pantograph type via Chebyshev polynomials," Communications in Nonlinear Science and Numerical Simulation, vol. 17, no. 12, pp. 4815-4830, 2012.

[25] E. Tohidi, A. H. Bhrawy, and Kh. Erfani, "A collocation method based on Bernoulli operational matrix for numerical solution of generalized pantograph equation," Applied Mathematical Modelling, vol. 37, pp. 4283-4294, 2013.

[26] I. Ali, H. Brunner, and T. Tang, "Spectral methods for pantograph-type differential and integral equations with multiple delays," Frontiers of Mathematics in China, vol. 4, no. 1, pp. 49-61, 2009.

[27] D. Trif, "Direct operatorial tau method for pantograph-type equations," Applied Mathematics and Computation, vol. 219, no. 4, pp. 2194-2203, 2012.

[28] R. Sakthivel, C. Chun, and J. Lee, "New travelling wave solutions of burgers equation with finite transport memory," Zeitschrift fur Naturforschung A, vol. 65, no. 8, pp. 633-640, 2010.

[29] R. Sakthivel, "Robust stabilization the Korteweg-de VriesBurgers equation by boundary control," Nonlinear Dynamics, vol. 58, no. 4, pp. 739-744, 2009.

[30] K. Pandey, L. Verma, and A. K. Verma, "Du Fort-Frankel finite difference scheme for Burgers equation," Arabian Journal of Mathematics, vol. 2, no. 1, pp. 91-101, 2013.

[31] Z.-Z. Sun and X.-N. Wu, "A difference scheme for Burgers equation in an unbounded domain," Applied Mathematics and Computation, vol. 209, no. 2, pp. 285-304, 2009.

[32] R. C. Mittal and R. Jiwari, "A differential quadrature method for numerical solutions of Burgers-type equations," International Journal of Numerical Methods for Heat \& Fluid Flow, vol. 22, no. 6-7, pp. 880-895, 2012.
[33] D. Rostamy and K. Karimi, "Hypercomplex mathematics and HPM for the time-delayed Burgers equation with convergence analysis," Numerical Algorithms, vol. 58, no. 1, pp. 85-101, 2011.

[34] A. G. Kudryavtsev and O. A. Sapozhnikov, "Determination of the exact solutions to the inhomogeneous burgers equation with the use of the darboux transformation," Acoustical Physics, vol. 57, no. 3, pp. 311-319, 2011.

[35] H. Caglar and M. F. Ucar, "Non-polynomial spline method for the solution of non-linear Burgers equation," Chaos and Complex Systems, pp. 213-218, 2013.

[36] M. Wang and F. Zhao, "Haar Wavelet method for solving twodimensional Burgers' equation," in Proceedings of the 2nd International Congress on Computer Applications and Computational Science, Advances in Intelligent and Soft Computing, vol. 145, pp. 381-387, 2012.

[37] J. Zhang, P. Wei, and M. Wang, "The investigation into the exact solutions of the generalized time-delayed Burgers-Fisher equation with positive fractional power terms," Applied Mathematical Modelling. Simulation and Computation for Engineering and Environmental Systems, vol. 36, no. 5, pp. 2192-2196, 2012.

[38] S. Rendine, A. Piazza, and L. L. Cavalli-Sforza, "Simulation and separation by principle components of multiple demic expansions in Europe," The American Naturalist, vol. 128, pp. 681-706, 1986.

[39] E. S. Fahmy, H. A. Abdusalam, and K. R. Raslan, "On the solutions of the time-delayed Burgers equation," Nonlinear Analysis. Theory, Methods \& Applications, vol. 69, no. 12, pp. 4775-4786, 2008.

[40] A. J. M. Jawad, M. D. Petković, and A. Biswas, "Soliton solutions of Burgers equations and perturbed Burgers equation," Applied Mathematics and Computation, vol. 216, no. 11, pp. 3370-3377, 2010.

[41] H. Kim and R. Sakthivel, "Travelling wave solutions for timedelayed nonlinear evolution equations," Applied Mathematics Letters, vol. 23, no. 5, pp. 527-532, 2010.

[42] E. H. Doha and A. H. Bhrawy, "An efficient direct solver for multidimensional elliptic Robin boundary value problems using a Legendre spectral-Galerkin method," Computers \& Mathematics with Applications, vol. 64, no. 4, pp. 558-571, 2012.

[43] E. H. Doha, W. M. Abd-Elhameed, and M. A. Bassuony, "New algorithms for solving high evenorder differential equations using third and fourth Chebyshev-Galerkin methods," Journal of Computational Physics, vol. 236, pp. 563-579, 2013.

[44] E. H. Doha and A. H. Bhrawy, "A Jacobi spectral Galerkin method for the integrated forms of fourth-order elliptic differential equations," Numerical Methods for Partial Differential Equations, vol. 25, no. 3, pp. 712-739, 2009.

[45] E. H. Doha, A. H. Bhrawy, D. Baleanu, and S. S. Ezz-Eldien, "On shifted Jacobi spectral approximations for solving fractional differential equations," Applied Mathematics and Computation, vol. 219, no. 15, pp. 8042-8056, 2013.

[46] R. Naz, I. Naeem, and F. M. Mahomed, "First integrals for two linearly coupled nonlinear Duffing oscillators," Mathematical Problems in Engineering, vol. 2011, Article ID 831647, 14 pages, 2011.

[47] M. Asadzadeh, D. Rostamy, and F. Zabihi, "Discontinuous Galerkin and multiscale variational schemes for a coupled damped nonlinear system of Schrodinger equations," Numerical Methods for Partial Differential Equations, 2013.

[48] R. A. Van Gorder and K. Vajravelu, "A general class of coupled nonlinear differential equations arising in self-similar solutions 
of convective heat transfer problems," Applied Mathematics and Computation, vol. 217, no. 2, pp. 460-465, 2010.

[49] M. Huang and Z. Zhou, "Standing wave solutions for the discrete coupled nonlinear Schrodinger equations with unbounded potentials," Abstract and Applied Analysis, vol. 2013, Article ID 842594, 6 pages, 2013. 


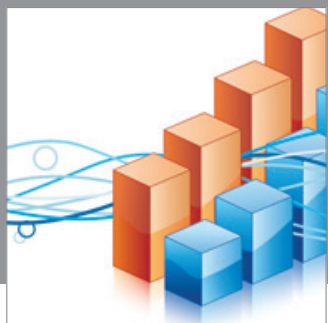

Advances in

Operations Research

mansans

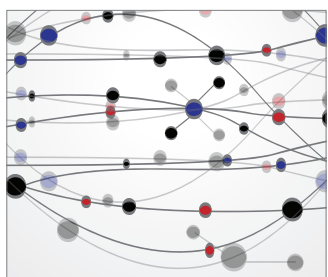

The Scientific World Journal
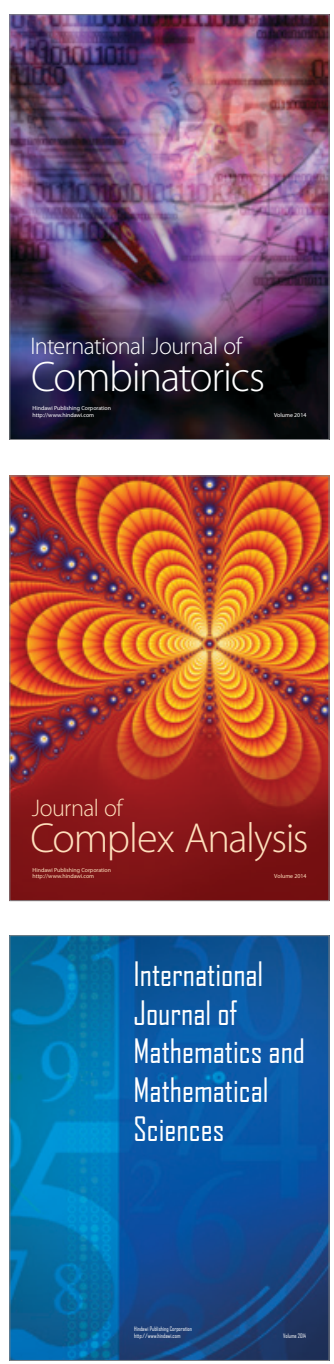
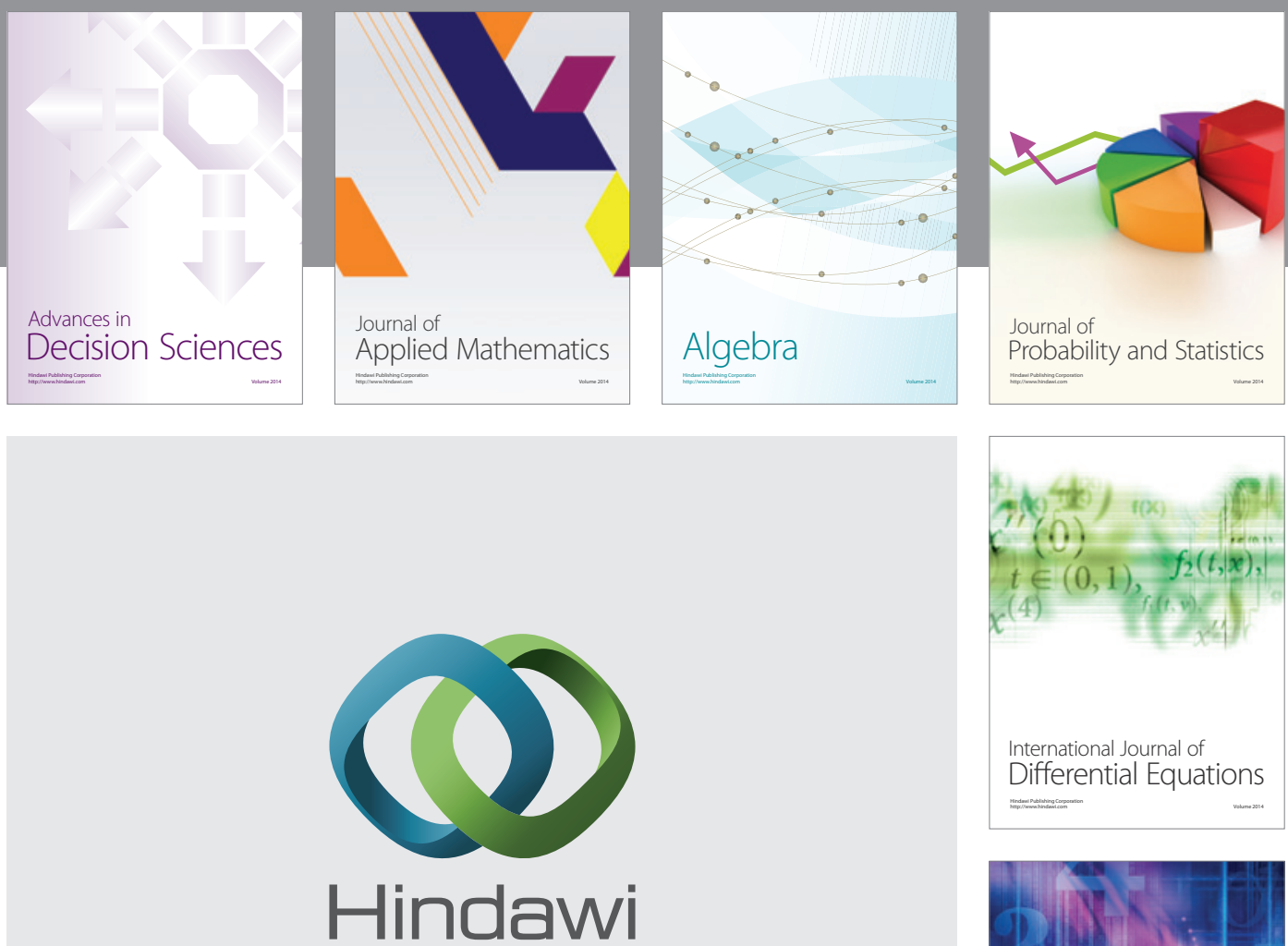

Submit your manuscripts at http://www.hindawi.com
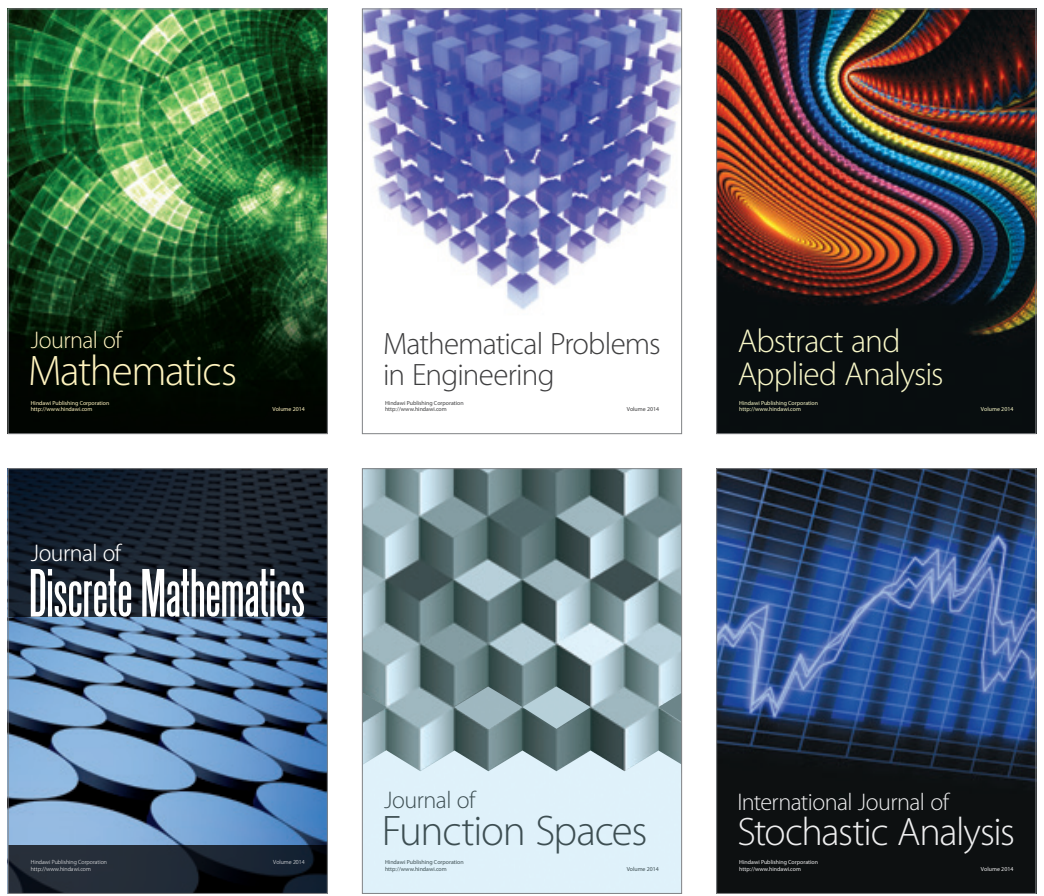

Journal of

Function Spaces

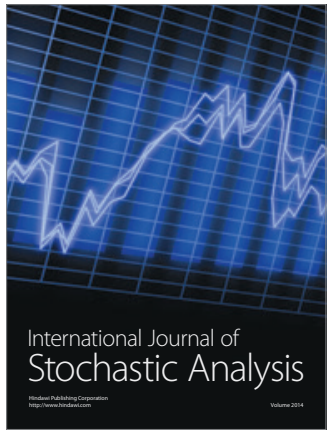

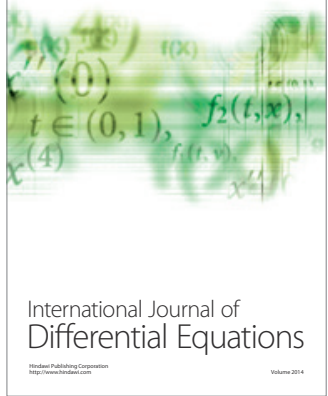
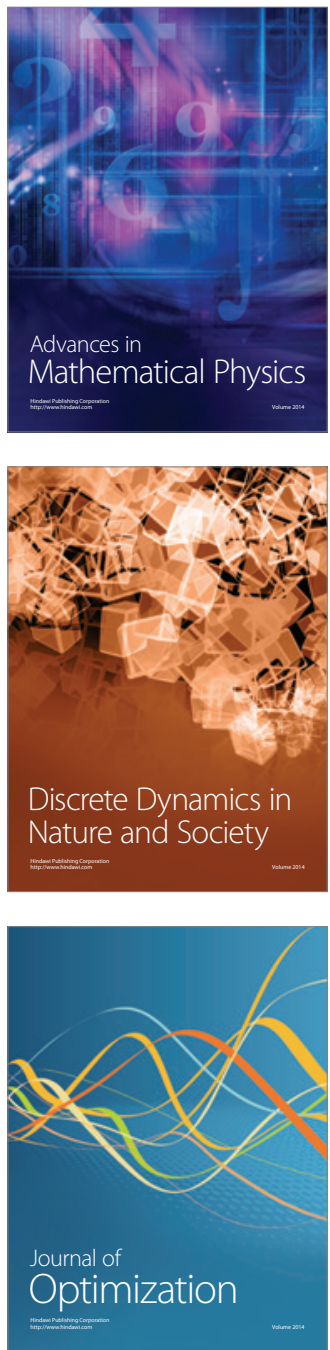